The stigma surrounding intimate partner violence: A cluster analysis study

By: Christine E. Murray, Allison Crowe, and Jason Brinkley

Murray, C. E., Crowe, A., \& Brinkley, J. (2015). The stigma surrounding intimate partner

violence: A cluster analysis study. Partner Abuse, 6(3), 320-336.

Made available courtesy of Springer Publishing Company: $\underline{\text { http://dx.doi.org/10.1891/1946- }}$ $\underline{6560.6 .3 .320}$

(C) 2015 Springer Publishing Company.

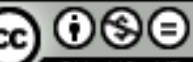

EY NO ${ }_{N D}$ This work is licensed under a Creative Commons AttributionNonCommercial-NoDerivatives 4.0 International License.

Abstract:

Existing research suggests that a significant stigma surrounds intimate partner violence, and this stigma can make it difficult for survivors to receive help. This article presents the results of a research study that used hierarchical cluster analysis, to identify whether certain types of stigma are more likely to co-occur. Survey results revealed four clusters based on participants' stigmarelated experiences: low stigma, blamed and black sheep, shame and separation, and high stigma. Participants in the high stigma group reported the highest levels of verbal abuse. Implications for theory, research, and practice are discussed.

Keywords: intimate partner violence $\mid$ domestic violence $\mid$ stigma $\mid$ cluster analysis

\title{
Article:
}

Intimate partner violence (IPV) describes "any form of physical, sexual, emotional, psychological, and/or verbal abuse between [current or former] partners in an intimate relationship" (Murray \& Graves, 2012, p. 14). The Centers for Disease Control and Prevention (2013) views IPV as a major public health problem. The potential negative consequences of IPV pose significant challenges for individual victims, child witnesses, and the broader society. For victims, these consequences may include mental and physical health symptoms as well as negative career and economic outcomes (Murray \& Graves, 2012). The potential consequences for child witnesses include anxiety and depression, behavioral problems, and difficulties in peer relationships (Stiles, 2002). According to the National Center for Injury Prevention and Control (2003), the annual costs of IPV on the U.S. economy are more than $\$ 5.8$ billion, including more than $\$ 4$ billion in costs for physical and mental health care and almost $\$ 0.9$ billion in lost work productivity.

The costs of IPV are high, and the needs of victims are critical. And yet, there is a significant stigma surrounding IPV that contributes to a lack of public awareness of the issue, makes it more difficult for survivors to receive help, and can hinder the recovery process for survivors (Crowe $\&$ Murray, 2015; Overstreet \& Quinn, 2013). Previously, researchers have documented the 
negative and/or stereotypical attitudes that many individuals hold toward the topic of IPV and the people who have experienced it (e.g., Brosi \& Rolling, 2010; Humphreys, 2008; Merritt-Gray \& Wuest, 1995). However, only recently have researchers begun to examine the construct of stigma to experiences of IPV. For example, Overstreet and Quinn (2013) presented a literature-based model of the stigma surrounding IPV, and two of the current authors (Crowe \& Murray, 2015) conducted a qualitative study examining ways that survivors experienced stigma from professionals who were in positions to help them.

The purpose of this study is to build on this new line of research examining the factors that underlie the stigma surrounding IPV. In the next section, we expand on the definition of stigma. Then, we review previous theoretical and research conceptualizations of the stigma surrounding IPV. Next, we discuss the methodology and results of a hierarchical cluster analysis study using data from a survey of 343 survivors of abusive relationships. The findings are then used to inform future theory, research, and practice regarding the stigma surrounding IPV.

\section{THE DEFINITION OF STIGMA}

The construct of stigma has been studied for decades by researchers across social science disciplines (Link \& Phelan, 2001). Link and Phelan (2001) offered a five-component conceptualization of stigma designed to present a cohesive definition of the term. The five components of stigma they outlined are as follows: (a) A label is placed on differences between people; (b) the labels are associated with negative stereotypes about the characteristics of people with those labels; (c) people create a sense of separation between themselves and those with the label (i.e., "separation of 'us' from 'them"'; Link \& Phelan, 2001, p. 367); (d) the people who are labeled experience diminished status and discrimination from others; and (e) people with the stigmatized label are denied access to "social, economic, and political power" (Link \& Phelan, 2001, p. 367). As such, stigma can be viewed as a process that occurs among groups of people and results in negative outcomes for the stigmatized groups.

Researchers have applied the concept of stigma to various groups-such as individuals who are HIV-positive (Davis, 2012) and those who have been diagnosed with a mental illness (Byrne, 2000). Therefore, it appears to be a process that disempowers many groups and holds common features across groups. However, the stigma that each unique group faces can look different from other groups (Link \& Phelan, 2001). In addition, social messages that are presented about various groups-such as through the media-can impact the nature of the stigma faced by members of the group. For example, when a mass shooting occurs and the gunman had a documented mental illness, the shooter's mental health often becomes a subject of the media coverage of the story (Szalavitz, 2012). This can lead media viewers to become more fearful of those with mental health conditions, further adding to the separation and discrimination of this group. Therefore, although some common processes are involved in the stigmatization of various groups, it is important to consider the unique aspects of stigma that different groups face.

\section{THE STIGMA SURROUNDING INTIMATE PARTNER VIOLENCE}

Overstreet and Quinn's (2013) IPV stigmatization model provided the first comprehensive application of the concept of stigma to IPV. These authors reviewed literature that supports the 
three components of stigma included in their model: (a) internalized stigma, (b) anticipated stigma, and (c) cultural stigma. Each of these components represents the different levels at which IPV stigmatization can occur: individual, interpersonal, and societal. Internalized stigma refers to "the extent to which people internalize negative IPV beliefs" (Overstreet \& Quinn, 2013, p. 117). Some of the self-perceptions that are associated with stigma internalization as found in the literature included self-blame, shame, embarrassment, guilt, and low self-esteem (Overstreet \& Quinn, 2013). Anticipated stigma describes "the degree to which people fear or expect stigmatization . . . if others know about their experiences" (Overstreet \& Quinn, 2013, p. 112). Some of the ways that anticipated stigma impacts victims of IPV include by believing that their friends and family members will not want to help them, by fearing negative outcomes if their abuse experiences were to become known in their workplaces, and by predicting that health care professionals will judge them upon disclosure of their abuse (Overstreet \& Quinn, 2013). Finally, cultural stigma describes "societal ideologies that delegitimize people who experience IPV" (Overstreet \& Quinn, 2013, p. 118). Cultural attitudes that can contribute to IPV stigmatization include judgment, blaming, minimizing the extent of the problem, and stereotypes about the types of people who are abused (Overstreet \& Quinn, 2013). Together, these components can make it more difficult for victims to seek help, although this can vary based on the extent to which survivors view the IPV as central to their identities and salient in their lives (Overstreet \& Quinn, 2013).

The IPV stigmatization model (Overstreet \& Quinn, 2013) provides a useful framework for understanding the different levels at which IPV survivors may experience stigma. However, the model is focused primarily on the source of the stigma (i.e., internal, relational, or societal). An additional layer to conceptualizing stigma involves the nature or type of stigma that survivors experience. Our previous research (Crowe \& Murray, 2015) suggests that there are different types of stigma that could occur at each of those levels. Using data derived from the same studies described in this article, the authors conducted a qualitative study to examine the types of stigma that IPV survivors experienced from specific sources (e.g., mental health professionals, attorneys, health care professionals, the police, the court system, the victims' workplaces, parenting-related resources, friends, family members, domestic violence agencies). The types of stigma that the survivors had faced fell into the following categories: (a) blame, or being viewed as responsible for the abuse; (b) discrimination, or being treated in prejudicial and/or stereotyped ways; (c) loss of status, or losing standing or perceived value related to their abuse; (d) isolation, or separation from others; (e) shame, experienced as guilt or self-blame; (f) being dismissed or denied, such as when the survivor's experiences with abuse were not viewed as serious or believable; and (g) blatant unprofessionalism, or when professionals from whom they sought help crossed professional boundaries and acted in unethical ways.

Ultimately, both conceptual frameworks described earlier (i.e., Crowe \& Murray, 2015; Overstreet \& Quinn, 2013) provide useful frameworks for understanding and addressing the stigma surrounding IPV. In fact, recently, these research groups have begun collaborating to develop an overarching model that integrates the two frameworks to provide a model of IPV stigma that accounts for both the source and type of stigma in one framework. As an additional precursor toward that ongoing work, this study used hierarchical cluster analysis to identify which types of stigma are most likely to co-occur as well as to identify whether there are patterns in the types of stigma that are associated with different types of abuse. Thus, the purpose of this 
study was to examine further the extent to which the concept of stigma as defined in social science literature applied to the lived experiences of survivors of abusive intimate relationships. The research questions that guided this study were as follows:

Research Question 1: Which types of stigma are most likely to co-occur?

Research Question 2: Do survivors' experiences of stigma differ based on whether they experienced different forms of abuse (i.e., physical, emotional/verbal, and/ or sexual)?

\section{METHOD}

The data analyzed for this study were collected as part of a larger series of mixed-methodology studies on the topic of IPV-related stigma with samples of survivors of past abusive relationships. The methodology described herein relates only to the specific research questions guiding this study. The data used for this study are derived from a subset set of questions from a larger electronic survey on the stigma surrounding IPV.

\section{Participants and Recruitment}

This study was open to individuals who (a) were at least 21 years of age, (b) were formerly abused by an intimate relationship partner (e.g., a boyfriend/girlfriend, life partner, spouse), (c) were out of any abusive relationship for at least 2 years, and (d) spoke English. Participants were required to pass an eligibility questionnaire before they could access the main survey.

Participants not meeting these eligibility criteria were excluded from this study. We implemented the minimum 2-year time frame for having been out of any abusive relationships to ensure that participants were not in an immediate crisis related to their abusive relationship. We used the minimum age limit of 21 years as an additional step to reduce the risk of participating in this study contributing to emotional distress because we assumed participants aged 21 years and older would have a suitable level of self-awareness to monitor their emotions while completing the survey instrument. The study was approved by two universities' institutional review boards before participant recruitment began.

The final sample should be considered a convenience sample, and participants were recruited in various ways. All recruitment strategies were done electronically and included e-mails to personal contacts, distributing a recruitment e-mail over relevant listserves, and posting notices about the survey on electronic discussion boards and Facebook pages that were relevant to the target population. Discussion boards and Facebook pages were identified by searching within Facebook and via Internet search engines using the following search terms: dating violence, domestic violence, intimate partner violence, sexual assault, abuse survivors, and trauma survivors. For Facebook pages, we posted the notice only when the site permitted "timeline" posts by any Facebook user (i.e., a person does not need to be a member of a group to post on the wall). For discussion boards, we only posted the notice when the board was public (i.e., one does not need to join to post to the discussion boards). As an incentive for participation, we held a drawing for one of two $\$ 50$ store gift cards for randomly selected participants who completed the survey. To keep participants' identities completely separate from their responses to the anonymous survey, participants who wished to enter the gift card drawing were directed to send an e-mail to a private e-mail address. 
There were 403 participants' survey responses included in the initial dataset for this study. However, because of incomplete responses, 60 observations were excluded from statistical analyses. This resulted in a sample of 343 participants whose data were included in the final analyses. The vast majority $(n=263,97.4 \%)$ of participants were female. The average age of participants was 39.7 years $(\mathrm{SD}=10.4)$. Participants' ethnicities/racial backgrounds were as follows: White $(\mathrm{n}=218,63.6 \%)$, African American/Black $(\mathrm{n}=27,7.9 \%)$, Hispanic/Latino/Latina ( $\mathrm{n}=26,7.6 \%)$, Native American $(\mathrm{n}=9,2.6 \%)$, Asian/Asian American ( $\mathrm{n}$ $=4,1.2 \%)$, and other $(\mathrm{n}=12,3.5 \%)$. Note that some participants did not report their racial/ethnic backgrounds. Most $(n=235,71.4 \%)$ participants reported that they have children. Regarding their highest level of completed education, 140 (40.8\%) participants reported having a bachelor's degree or graduate degree. Participants had diverse household income levels, with 116 participants (42.5\%) having incomes less than $\$ 30,000,84$ participants $(30.8 \%)$ with incomes between $\$ 30,000$ and \$59,000, $48(17.6 \%)$ between $\$ 60,000$ and $\$ 100,000$, and $25(9.2 \%)$ participants with incomes more than $\$ 100,000$. Based on the eligibility criteria for this study, all participants had been out of any abusive relationships for at least 2 years. The average number of years participants reported having been out of their most recent abusive relationships was 7.6 years $(\mathrm{SD}=7.2)$, with responses ranging from 2 to 40 years.

Instrumentation

The data analyzed for this study were collected as part of a larger Internet-based survey on the stigma surrounding IPV. The survey was hosted on the Internet-based survey software, Qualtrics, which is located on a secure server. The survey included five parts: (a) a demographic questionnaire, (b) questions about participants' past experiences within abusive relationships, (c) a section on the types of stigma that participants experienced (which was the focus of this study and will be described further in the following text), (d) questions about the sources of the stigma they experienced, and (e) open-ended questions about whether and how participants overcame the stigma surrounding abuse.

A single, researcher-created question was used to measure the type(s) of abuse that participants had experienced. As part of a series of questions on participants' experiences in their most recent relationship that included any form of IPV, participants were asked, "What forms of abuse would you say that you experienced in this relationship? (Check all that apply)." The response options, from which participants were permitted to select as many options as possible, included the following: physical abuse, emotional abuse, verbal abuse, sexual abuse, and other. (Note: Participants selecting "other" were asked to specify what other forms of abuse they experienced.) Although lengthier assessment instruments are published to measure experiences with abuse, for this study, we were most interested in learning about participants' general experiences with abuse and chose this assessment approach as a practical approach to categorizing participants' experiences with different types of abuse. In addition, our goal was to gain a more in-depth understanding of participants' experiences with abuse, and therefore we included additional open-ended questions asking participants to describe their experiences with IPV. This approach also was consistent with our aim to understand the unique experiences that survivors have with abuse. 
For the section on types of stigma on which this study is based, participants were asked to rate their stigma-related experiences based on a 5-point Likert-type scale ( $1=$ does not apply at all; 2 = applies a little; $3=$ somewhat applies $; 4=$ mostly applies; $5=$ completely applies $)$. The researchers created this part of the survey based on a review of existing literature on stigma, focusing particularly on Link and Phelan's (2001) five-component conceptualization of stigma. It was necessary to create this assessment format for this study because there is currently no other published measure of the stigma surrounding IPV.

Participants were provided with the following introductory text:

As you know from the informed consent document, we are interested in studying the concept of stigma and how well it applies to the experiences of survivors of intimate partner violence (IPV). Stigma has been defined as "a product of disgrace that sets a person apart from others" (Byrne, 2000). Various researchers have hinted that there is a strong stigma surrounding these experiences, but we have not been able to find any research that directly studies this issue by getting input from survivors themselves. This survey is the second part of our research on this topic. Previously, we interviewed a number of survivors of IPV to ask about their experiences with stigma, and the current study is designed to learn about these experiences from a larger, more diverse sample. Below you will find several terms that have been used to describe the experience of stigma or being stigmatized. To what extent do each of these terms apply to your experience of abuse? Below, please provide an additional explanation about your experiences for any items you rate as 3 or above.

The terms that participants were asked to rate were as follows: (a) blame, (b) black sheep of the family role, (c) discrimination, (d) isolation, (e) being labeled, (f) loss of power, (g) loss of status, (h) secrecy, (i) separation, (j) shame, (k) social exclusion, and (l) stereotypes. To provide additional depth to these ratings, for any items for which the participants rated 3 or higher on the Likert scale (i.e., the item at least somewhat applies), participants were asked to provide an openended description of their experiences related to that term. Because of the bulk of qualitative data provided, an analysis of participants' qualitative responses is beyond the scope of this article, and currently, an additional study is underway to analyze these qualitative data.

\section{Data Analyses}

A preliminary step to the data analysis was addressing missing data because several participants did not rate all 12 types of stigma presented on the survey. Given the nature of the information being collected, all questions were optional for participants to complete, and this resulted in seemingly coarsened data because many of the more sensitive questions on abuse and demographics were answered, but some participants did not answer some of the stigma questions. The investigators hypothesize that the set of missing data in the stigma items was an indicator that stigma was not a factor in the abusive relationship described. We believe that participants' failure to complete all responses may have been an artifact of the fact that some of the preceding sections of the survey had a "check all that apply" format, such that on those items, participants simply did not check responses when they did not apply to their situations. Thus, in accounting for missing data in the subsequent data analyses, we coded missing responses as 1 
(does not apply at all). We present evidence in a later section that suggests that the assumption about the nature of the missing data is tenable.

To facilitate data analyses and interpretation of the cluster analysis findings, the participants' ratings on the stigma-type items were organized into three ordinal groups, designed to represent low-level experiences of that type of stigma (Group $1=$ missing or 1 [does not apply at all]), moderate levels of stigma (Group $2=2$ [ applies a little ] or 3 [ somewhat applies ]), and high levels of stigma (Group $3=4$ [mostly applies] or $=$ [completely applies]). In this way, each observation's responses on the 12 stigma items were recoded into 12 new variables, with the mentioned ordinal groupings for each response.

To explore whether certain types of the 12 types of stigma appear to co-occur more frequently, a hierarchical cluster analysis was performed on the recoded data. Several different cluster techniques (including centroid method, Ward's method, single linkage, and complete linkage) and solutions were explored, and a four-cluster solution was chosen (using Ward's method) that seemed to best represent the associations in the types of stigma as well as account for the pattern of missing data described earlier. A series of Fisher's exact tests were used to explore associations between stigma cluster groups and types of abuse participants had experienced. A common significance level of .05 was used for all hypotheses testing. All analyses were performed using JMP Pro 11 software.

\section{RESULTS}

Among the 343 respondents, 53\% reported that they had experienced physical abuse, $66 \%$ experienced emotional abuse, $61 \%$ experienced verbal abuse, $40 \%$ experienced sexual abuse, and $15 \%$ experienced some other form of abuse. (Note: Participants could have experienced multiple forms of abuse, and therefore the sum of percentages is greater than $100 \%$.)

Table 1 presents the percentages of participants' ratings for each type of stigma, before these ratings were collapsed into the three groups representing low, moderate, and high levels of stigma. Note that the distribution of missing data is different across the 12 stigma types. It is also worth noting that although between $33 \%$ and $39 \%$ of each question has missing responses, much of the data with missing responses were missing only for particular items. In addition, all 343 individuals answered survey questions that appeared after the questions on stigma. Taken together, the evidence suggests that there may have been some coarsening (unintended missingness because of the data collection method) of the data.

In addition to nonuniformity in the missing stigma questions, there were observed associations between emotional and verbal abuse rates and nonresponse in stigma questions. In Table 2 in the following text, the nonresponse rates for each stigma item is separated between those that have and have not experienced either emotional or verbal abuse. Fisher's exact test illustrates that there are significant associations between the rates of abuse and having a response in stigmabased categories. Simply put, individuals who did not experience emotional and verbal abuse demonstrated higher rates of nonresponse in the stigma categories, which again agrees with the hypothesis that those who did not experience such stigma chose to leave those questions blank. 
TABLE 1. Percentage of Participants' Ratings for Levels of Each Type of Stigma

\begin{tabular}{|lcccccc|}
\hline Type of Stigma & Missing & $\begin{array}{c}\text { 1-Does Not } \\
\text { Apply at All }\end{array}$ & $\begin{array}{c}\text { 2-Applies a } \\
\text { Little }\end{array}$ & $\begin{array}{c}\text { 3-Somewhat } \\
\text { Applies }\end{array}$ & $\begin{array}{c}\text { 4-Mostly } \\
\text { Applies }\end{array}$ & $\begin{array}{c}\text { 5-Completely } \\
\text { Applies }\end{array}$ \\
\hline Blame & $38.78 \%$ & $5.54 \%$ & $11.08 \%$ & $11.37 \%$ & $13.12 \%$ & $20.12 \%$ \\
Black sheep & $38.48 \%$ & $22.45 \%$ & $12.54 \%$ & $7.00 \%$ & $5.83 \%$ & $13.70 \%$ \\
Discrimination & $38.78 \%$ & $24.78 \%$ & $13.99 \%$ & $8.16 \%$ & $6.41 \%$ & $7.87 \%$ \\
Isolation & $38.78 \%$ & $4.96 \%$ & $5.25 \%$ & $8.16 \%$ & $12.83 \%$ & $30.03 \%$ \\
Labeled & $37.32 \%$ & $9.62 \%$ & $11.95 \%$ & $11.08 \%$ & $12.83 \%$ & $17.20 \%$ \\
Loss of power & $38.48 \%$ & $5.51 \%$ & $6.41 \%$ & $7.00 \%$ & $10.79 \%$ & $31.78 \%$ \\
Loss of status & $33.53 \%$ & $13.12 \%$ & $10.79 \%$ & $10.20 \%$ & $10.50 \%$ & $21.87 \%$ \\
Secrecy & $34.11 \%$ & $4.96 \%$ & $5.83 \%$ & $6.12 \%$ & $11.08 \%$ & $37.90 \%$ \\
Separation & $33.82 \%$ & $6.12 \%$ & $7.87 \%$ & $9.04 \%$ & $8.16 \%$ & $34.99 \%$ \\
Shame & $33.82 \%$ & $3.79 \%$ & $5.54 \%$ & $8.16 \%$ & $9.91 \%$ & $38.78 \%$ \\
Social exclusion & $33.24 \%$ & $7.58 \%$ & $6.71 \%$ & $10.79 \%$ & $12.24 \%$ & $29.45 \%$ \\
Stereotype & $34.40 \%$ & $10.79 \%$ & $13.41 \%$ & $8.75 \%$ & $10.79 \%$ & $21.87 \%$ \\
\hline
\end{tabular}

Table 2 also illustrates that the missingness in the stigma items is not at random. By illustrating differences in the response rates between those who have and have not experienced particular forms of abuse rules out the use of common missing data methods such as multiple imputation analyses. Finding such discrepancies within the data violates the traditional missing at random assumption necessary for many standard methods (Tsiatis, 2006). However, taking the two previous tables together, a viable approach for dealing with the missing data was to recode the data as previously stated into three ordinal groups with the missing values and lowest end observations forming the lowest subgroup.

TABLE 2. Nonresponse Rates for Each Stigma Item Based on Emotional and Verbal Abuse Experiences

\begin{tabular}{|lcccccccc|}
\hline & \multicolumn{4}{c}{ Emotional } & \multicolumn{4}{c|}{ Verbal } \\
\cline { 2 - 8 } \multicolumn{1}{c}{} & No & Yes & Difference & $\boldsymbol{p}$ Value $^{\mathbf{a}}$ & No & Yes & Difference & $\boldsymbol{p}$ Value $^{\mathbf{a}}$ \\
\hline Blame & $48.3 \%$ & $33.8 \%$ & $14.5 \%$ & .0103 & $47.8 \%$ & $33.0 \%$ & $14.8 \%$ & .0066 \\
Black sheep & $49.2 \%$ & $32.9 \%$ & $16.3 \%$ & .0036 & $48.5 \%$ & $32.1 \%$ & $16.5 \%$ & .0030 \\
Discrimination & $49.2 \%$ & $33.3 \%$ & $15.8 \%$ & .0051 & $48.5 \%$ & $32.5 \%$ & $16.0 \%$ & .0044 \\
Isolation & $49.2 \%$ & $33.3 \%$ & $15.8 \%$ & .0051 & $48.5 \%$ & $32.5 \%$ & $16.0 \%$ & .0044 \\
Labeled & $45.8 \%$ & $32.9 \%$ & $12.9 \%$ & .0253 & $44.0 \%$ & $33.0 \%$ & $11.0 \%$ & .0515 \\
Loss of power & $46.6 \%$ & $34.2 \%$ & $12.4 \%$ & .0270 & $44.8 \%$ & $34.5 \%$ & $10.3 \%$ & .0686 \\
Loss of status & $35.6 \%$ & $32.4 \%$ & $3.2 \%$ & .6303 & $36.6 \%$ & $31.6 \%$ & $5.0 \%$ & .3505 \\
Secrecy & $36.4 \%$ & $32.9 \%$ & $3.6 \%$ & .0939 & $37.3 \%$ & $32.1 \%$ & $5.3 \%$ & .0472 \\
Separation & $39.8 \%$ & 30.7 & $9.2 \%$ & .0939 & $40.3 \%$ & $29.7 \%$ & $10.6 \%$ & .0472 \\
Shame & $39.0 \%$ & $31.1 \%$ & $7.9 \%$ & .1510 & $38.8 \%$ & $30.6 \%$ & $8.2 \%$ & .1292 \\
Social exclusion & $39.0 \%$ & $30.2 \%$ & $8.8 \%$ & .1170 & $38.8 \%$ & $29.7 \%$ & $9.1 \%$ & .0998 \\
Stereotype & $39.0 \%$ & $32.0 \%$ & $7.0 \%$ & .0998 & $38.8 \%$ & $31.6 \%$ & $7.2 \%$ & .2001 \\
\hline
\end{tabular}

${ }^{a} p$ values are the result of a two-sided Fisher's exact test.

The results of the cluster analysis are listed in Table 3. As demonstrated in the table, 50\% was the cutoff for including a particular type of stigma as falling into a stigma grouping (i.e., low/moderate/high). The cluster sizes were rather robust, with the smallest group containing 47 observations. The following is a summary of the description of the groupings, along with our interpretation and label for each cluster: 
Cluster 1. Cluster 1 is labeled the low stigma group. This cluster is a clear grouping of 86 individuals who report virtually no stigma, with between two-thirds and $98 \%$ of stigma items responded as either missing or a value of 1 .

Cluster 2. Cluster 2 is labeled the blamed and black sheep group. This group demonstrated high levels of stigma on only two variables, blame and the "black sheep of the family role," yet they also reported low levels of stigma on most other types of stigma.

Cluster 3. Cluster 3 is labeled the shame and separation group. This group rated five types of stigma as applying to their experiences at a high level: secrecy, separation, shame, social exclusion, and stereotyping. However, they described themselves as not experiencing nearly all of the other forms of stigma.

Cluster 4. Cluster 4 appears to be the cluster of most interest, and it was labeled the high stigma group. Herein is a subgroup of 146 individuals who report higher levels of stigma across all but four types of stigma. A deeper analysis revealed that all 146 individuals list an original ranking of 4 or 5 for at least one of each of these stigma items. In fact, 136 of the 146 individuals (93\%) coded a Level 5 for at least one type of stigma.

TABLE 3. Hierarchical Cluster Analysis Groupings

\begin{tabular}{|c|c|c|c|c|}
\hline \multicolumn{2}{|l|}{ Group } & 1 & 2 & 3 \\
\hline \multirow{12}{*}{$\begin{array}{l}\text { Cluster } 1(n=86) \\
\text { Low stigma }\end{array}$} & Blame & $87 \%$ & $12 \%$ & $1 \%$ \\
\hline & Black sheep & $95 \%$ & $3 \%$ & $1 \%$ \\
\hline & Discrimination & $98 \%$ & $1 \%$ & $1 \%$ \\
\hline & Isolation & $87 \%$ & $12 \%$ & $1 \%$ \\
\hline & Labeled & $67 \%$ & $24 \%$ & $8 \%$ \\
\hline & Loss of power & $70 \%$ & $9 \%$ & $21 \%$ \\
\hline & Loss of status & $85 \%$ & $2 \%$ & $13 \%$ \\
\hline & Secrecy & $76 \%$ & $7 \%$ & $17 \%$ \\
\hline & Separation & $94 \%$ & $5 \%$ & $1 \%$ \\
\hline & Shame & $90 \%$ & $7 \%$ & $3 \%$ \\
\hline & Social exclusion & $97 \%$ & $2 \%$ & $1 \%$ \\
\hline & Stereotype & $97 \%$ & $2 \%$ & $1 \%$ \\
\hline \multirow{12}{*}{$\begin{array}{l}\text { Cluster } 2(n=47) \\
\text { Blamed and black sheep }\end{array}$} & Blame & $2 \%$ & $19 \%$ & $79 \%$ \\
\hline & Black sheep & $23 \%$ & $26 \%$ & $51 \%$ \\
\hline & Discrimination & $23 \%$ & $47 \%$ & $30 \%$ \\
\hline & Isolation & $2 \%$ & $4 \%$ & $94 \%$ \\
\hline & Labeled & $57 \%$ & $3 \%$ & $36 \%$ \\
\hline & Loss of power & $51 \%$ & $9 \%$ & $94 \%$ \\
\hline & Loss of status & $64 \%$ & $17 \%$ & $19 \%$ \\
\hline & Secrecy & $66 \%$ & $4 \%$ & $30 \%$ \\
\hline & Separation & $98 \%$ & $2 \%$ & $0 \%$ \\
\hline & Shame & $98 \%$ & $0 \%$ & $2 \%$ \\
\hline & Social exclusion & $100 \%$ & $0 \%$ & $0 \%$ \\
\hline & Stereotype & $100 \%$ & $0 \%$ & $0 \%$ \\
\hline \multirow{7}{*}{$\begin{array}{l}\text { Cluster } 3(n=64) \\
\text { Shame and serparation }\end{array}$} & Blame & $100 \%$ & $0 \%$ & $0 \%$ \\
\hline & Black sheep & $100 \%$ & $0 \%$ & $0 \%$ \\
\hline & Discrimination & $100 \%$ & $0 \%$ & $0 \%$ \\
\hline & Isolation & $97 \%$ & $2 \%$ & $2 \%$ \\
\hline & Labeled & $61 \%$ & $19 \%$ & $20 \%$ \\
\hline & Loss of power & $56 \%$ & $16 \%$ & $28 \%$ \\
\hline & Loss of status & $28 \%$ & $36 \%$ & $3 \%$ \\
\hline
\end{tabular}




\begin{tabular}{|llccc|}
\hline & Secrecy & $19 \%$ & $11 \%$ & $70 \%$ \\
& Separation & $2 \%$ & $22 \%$ & $77 \%$ \\
& Shame & $5 \%$ & $8 \%$ & $88 \%$ \\
& Social exclusion & $2 \%$ & $23 \%$ & $75 \%$ \\
& Stereotype & $13 \%$ & $34 \%$ & $53 \%$ \\
\hline Cluster 4 $(n=146)$ & Blame & $8 \%$ & $40 \%$ & $52 \%$ \\
High stigma & Black sheep & $36 \%$ & $36 \%$ & $29 \%$ \\
& Discrimination & $40 \%$ & $36 \%$ & $23 \%$ \\
& Isolation & $8 \%$ & $23 \%$ & $69 \%$ \\
& Labeled & $25 \%$ & $29 \%$ & $45 \%$ \\
& Loss of power & $21 \%$ & $16 \%$ & $62 \%$ \\
& Loss of status & $27 \%$ & $27 \%$ & $47 \%$ \\
& Secrecy & $18 \%$ & $18 \%$ & $64 \%$ \\
& Separation & $6 \%$ & $27 \%$ & $67 \%$ \\
& Shame & $2 \%$ & $25 \%$ & $73 \%$ \\
& Social exclusion & $6 \%$ & $29 \%$ & $64 \%$ \\
& Stereotype & $12 \%$ & $36 \%$ & $53 \%$ \\
\hline
\end{tabular}

Note. 1 = low-level stigma; 2 = moderate stigma; 3 = high stigma.

Participants were asked to indicate the forms of abuse they experienced in their past abusive relationship. They could check all that applied for this statement and were given the following response options: (a) physical abuse, (b) emotional abuse, (c) verbal abuse, (d) sexual abuse, and (e) other. Other responses were not included in this analysis. Table 4 illustrates the observed abuse rates across the four stigma-based clusters. Again, the statistical procedure used was Fisher's exact test. There was a significant association between verbal abuse and cluster groupings, with Cluster 4 (i.e., "high stigma") participants demonstrating significantly higher rates of verbal abuse as compared to Clusters 1,2 , and 3 . Although not statistically significant (p $=.0896$ ), a similar association appears to be likely for emotional abuse, with more Cluster 4 participants reporting emotional abuse than the other clusters. Differences in rates of physical abuse and sexual abuse were less pronounced among the four clusters.

TABLE 4. Differences in Types of Abuse Between Clusters

\begin{tabular}{|c|c|c|c|c|c|}
\hline & $\begin{array}{c}\text { Cluster 1: Low } \\
\text { Stigma }\end{array}$ & $\begin{array}{c}\text { Cluster 2: Blamed } \\
\text { and Black Sheep }\end{array}$ & $\begin{array}{l}\text { Cluster 3: Shame } \\
\text { and Separation }\end{array}$ & $\begin{array}{c}\text { Cluster 4: High } \\
\text { Stigma } \\
\end{array}$ & $p$ Value \\
\hline Physical Affirmative count & 41 & 26 & 27 & 86 & .1081 \\
\hline$\%$ & 48 & 55 & 42 & 59 & \\
\hline Emotional Affirmative count & 50 & 31 & 38 & 106 & .0896 \\
\hline$\%$ & 58 & 66 & 59 & 73 & \\
\hline Verbal Affirmative count & 47 & 27 & 32 & 103 & $.0133^{*}$ \\
\hline$\%$ & 55 & 57 & 50 & 71 & \\
\hline Sexual Affirmative count & 31 & 18 & 25 & 62 & .8127 \\
\hline$\%$ & 36 & 38 & 39 & 42 & \\
\hline
\end{tabular}

\section{DISCUSSION}

The findings of this study support the notion that many survivors of IPV face some form of stigma related to their experiences of abuse. The findings also suggest that the amount of stigma varies. It appears that some survivors report experiencing minimal levels of stigma (i.e., the "low stigma" group), and therefore stigma may not be a universal experience for all survivors. It is 
also possible that some survivors experience stigma but either do not label it as such or the terms included on the survey used in this study do not resonate with some survivors' experiences.

Two identified clusters (i.e., the "blamed and black sheep" and the "shame and separation") of participants in this study experienced more moderate levels of stigma. The associations among the identified types of stigma experienced within these groups offer insights to understanding how aspects of stigma may intersect for unique outcomes for survivors. For example, in Cluster 2 , the blamed and black sheep group, the experience of being and/or feeling blamed or somehow responsible for the abuse they experienced may contribute to feelings of not fitting in or being treated differently within their families and other social networks. Similarly, for Cluster 3, the compound experiences of secrecy, separation, shame, social exclusion, and stereotyping all may interact to contribute to survivors feeling negative emotions about how they are perceived and treated by others.

The largest identified cluster was the high stigma group, and although this group did endorse certain types of stigma less than others, across the board, they indicated that they experienced the highest levels of numerous types of stigma. It is noteworthy that this group also reported the highest rates of verbal abuse and emotional abuse, although the difference for emotional abuse was not statistically significant at the $p<.05$ level. This suggests that greater experiences of psychological abuse contribute to stronger perceptions of being stigmatized related to ones' abuse victimization. At the present, it remains unclear why sexual and physical abuse may not be linked as closely to experiences of stigma. We speculate that this may result from the process of stigma being internalized (Overstreet \& Quinn, 2013) more as a direct result of the verbal denigration and emotional toll associated with emotional and physical abuse. However, this remains an important area for future research.

\section{LIMITATIONS}

There are several limitations that must be considered in the interpretation of the findings of this study. First, the sample was a convenience sample, and although the sample size was relatively large, we cannot know the extent to which the findings of this study are able to be generalized to the broader population of survivors of IPV. Because the survey was online and all participant recruitment was done electronically, we can assume that another group of participants who is not as connected to technology may differ from this group of participants. Second, the survey instrument was created specifically for this study, and therefore there was no previous information about the instrument's psychometric properties. Third, the time-related eligibility criterion (i.e., participants had to have been out of any abusive relationship for at least 2 years) was implemented as a way to minimize the risk that participants would have experienced emotional distress upon completion of the study. However, it is possible that participants' perspectives on their stigma-related experiences have changed over time, and they may have viewed these same experiences differently in the immediate aftermath of their abuse. Fourth, in the introduction to the stigma-related terms that we asked participants to rate, we offered a brief overview of the concept of stigma. We provided this information to offer participants a standard description of stigma on which to base their ratings. However, it is possible that this text could have somehow biased participants' responses to the items they rated. Fifth, there were very few males, as compared to females, represented in the sample, so future research is needed to 
determine whether male participants' experiences of stigma related to IPV may differ from those of females. Finally, although we used a sound, standardized procedure for addressing missing data, it is not possible to determine the reason that participants chose not to respond to certain items, and it is unknown the extent to which the missing data could have skewed the findings.

\section{IMPLICATIONS FOR THEORY, RESEARCH, AND PRACTICE}

Currently, there is a need for additional theoretical and research attention to conceptualizing the nature of the stigma surrounding IPV. The findings of this study suggest a need for greater understanding of how the different aspects of stigma relate to one another. For example, scholars can work to advance theories that may help to understand why experiences of blame and being in the black sheep of the family role would be related to one another. Similarly, additional theoretical consideration should be paid to identifying factors that contribute to higher or lower levels of stigma experiences. The theoretical work by Overstreet and Quinn (2013) can help to guide this work because it may be that different sources of stigma contribute more to perceived stigma than others.

The association between higher rates of verbal abuse and higher experiences of stigma warrants further theoretical and research attention. Qualitative research may help to provide information about how these experiences are linked from the perspective of survivors. Quantitative research, likewise, can be useful to identify patterns of verbal abuse and stigma over time, including whether there are associations with other forms of abuse that were not identified in this study. Studying these processes longitudinally also could help to identify patterns of stigma that occur at different points in time (e.g., while in the abusive relationship, during the process of leaving, and at longer term follow-up periods). To strengthen future research, there is a need to develop a measurement instrument that could provide a more standardized way to assess IPV-related stigma experiences.

The findings of this study also have implications for professionals who work with clients who have experienced IPV victimization. In particular, this study identified a group of survivors of IPV victimization who faced high levels of stigma, and two other groups faced moderate levels of stigma related to their abuse. This suggests that professionals can work with survivors to identify whether and how they have faced, or are currently facing, stigmatizing experiences, which may make it more difficult for them to seek and receive help (Crowe \& Murray, 2015; Overstreet \& Quinn, 2013). Likewise, these stigma-related experiences may compound the traumatic effects of the abuse they experience. Therefore, professionals working with clients impacted by IPV should be mindful of the stigma that many survivors face, and they can work proactively to ensure that they provide services in nonstigmatizing contexts and are able to access needed resources available in the broader community.

In conclusion, this study examined experiences of stigma among a sample of survivors of IPV. The findings revealed that although a subset of the sample reported low levels of stigma, other survivors experienced moderate to high levels of stigma. Higher levels of stigma appear to be linked to greater experiences of verbal and emotional abuse. The results of this study support the need for further research to better understand the stigma surrounding IPV as well as the development of effective practice strategies for addressing the stigma and its impact on survivors 
at the individual and societal levels. Given the potential negative impacts of this stigma, efforts to prevent it, as well as to support survivors who have faced it, are in critical demand.

\section{REFERENCES}

Brosi, M. W., \& Rolling, E. S. (2010). A narrative journey for intimate partner violence: From victim to survivor. The American Journal of Family Therapy, 38, 237-250.

http://dx.doi/org/10.1080/01926180902961761

Byrne, P. (2000). Stigma of mental illness and ways of diminishing it. Advances in Psychiatric Treatment, 6, 65-72.

Centers for Disease Control and Prevention. (2013). Intimate partner violence. Retrieved from http:/www.cdc.gov/VIOLENCEPREVENTION/intimatepartnerviolence/

Crowe, A., \& Murray, C. E. (2015). Stigma from professional helpers toward survivors of intimate partner violence. Partner Abuse, 6(2), 157-179.

Davis, K. B. (2012). Understanding and addressing stigma and attachment insecurity in HIVpositive women who experience intimate partner violence: A model of medical and psychosocial care. Social Work in Health Care, 51(3), 213-231.

http://dx.doi.org/10.1080/00981389.2011.614835

Humphreys, C. (2008). Problems in the system of mandatory reporting of children living with domestic violence. Journal of Family Studies, 14, 228-239.

Link, B. G., \& Phelan, J. C. (2001). Conceptualizing stigma. Annual Review of Sociology, 27, $363-385$.

Merritt-Gray, M., \& Wuest, J. (1995). Counteracting abuse and breaking free: The process of leaving revealed through women's voices. Health Care for Women International, 16, 399-412. http://dx.doi.org/10.1080/07399339509516194

Murray, C. E., \& Graves, K. N. (2012). Responding to family violence: A comprehensive, research-based guide for therapists. New York, NY: Routledge.

National Center for Injury Prevention and Control. (2003). Costs of intimate partner violence against women in the United States. Atlanta, GA: Centers for Disease Control and Prevention. Retrieved from http://www.cdc.gov/violenceprevention/pdf/ipvbook-a.pdf

Overstreet, N. M., \& Quinn, D. M. (2013). The intimate partner violence stigmatization model and barriers to help seeking. Basic and Applied Social Psychology, 35, 109-122.

Stiles, M. M. (2002). Witnessing domestic violence: The effect on children. American Family Physician, 66, 2052-2067. 
Szalavitz, M. (2012, July). After Aurora, questions about mass murder and mental illness. Time Magazine. Retrieved from http://healthland.time.com/2012/07/31/mass-murder-and-mentalillness-the-interplay-of-stigma-culture-and-disease/

Tsiatis, A. A. (2006). Semiparametric theory and missing data. New York, NY: Springer.

Correspondence regarding this article should be directed to Christine E. Murray, $\mathrm{PhD}$, Department of Counseling and Educational Development, The University of North Carolina, 228 Curry Building, Greensboro, NC 27402. E-mail: cemurray@uncg.edu 